\title{
Equivalence of the Projected Forward Dynamics and the Dynamically Consistent Inverse Solution
}

\author{
João Moura*†, Vladimir Ivan $^{\dagger}$, Mustafa Suphi Erden*, and Sethu Vijayakumar ${ }^{\dagger}$ \\ *Institute of Sensors, Signals and Systems, Heriot-Watt University, Mountbatten Building, EH14 4AS, Edinburgh, UK \\ $\dagger$ Institute of Action, Perception, and Behaviour, University of Edinburgh, Crichton Street 10, EH8 9AB, Edinburgh, UK
}

\begin{abstract}
The analysis, design, and motion planning of robotic systems, often relies on its forward and inverse dynamic models. When executing a task involving interaction with the environment, both the task and the environment impose constraints on the robot's motion. For modeling such systems, we need to incorporate these constraints in the robot's dynamic model. In this paper, we define the class of Task-based Constraints (TbC) to prove that the forward dynamic models of a constrained system obtained through the Projection-based Dynamics (PbD), and the Operational Space Formulation (OSF) are equivalent. In order to establish such equivalence, we first generalize the OSF to a rank deficient Jacobian. This generalization allow us to numerically handle redundant constraints and singular configurations, without having to use different controllers in the vicinity of such configurations. We then reformulate the $\mathrm{PbD}$ constraint inertia matrix, generalizing all its previous distinct algebraic variations. We also analyse the condition number of different constraint inertia matrices, which affects the numerical stability of its inversion. Furthermore, we show that we can recover the operational space control with constraints from a multiple Task-based Constraint abstraction.
\end{abstract}

\section{INTRODUCTION}

Motion planning, control, learning, and state estimation, often rely on modelling a robot as a dynamical system. In the absence of interaction with the environment, we refer to the robot's motion as unconstrained, given that its state evolves solely according to its dynamics. The interaction with the environment imposes constraints on the dynamical system, in the form of contacts, rigid connections, tasks and behaviours. For example, consider a floating base robot, such as a humanoid (Fig. 1), carrying a jar of water. This robot needs to exploit the contact constraints for locomotion while maintaining balance without spilling the water. The latter are tasks that also constrain its dynamic motions. Another example is a robot with structural constraints, such as closed kinematic loops (Fig. 1). The same robot, might require a compliant behaviour towards safe human robot interaction, which also constrains the dynamical motion of the robot. Our motivation is to model dynamical systems with a generic class of constraints that are useful in developing motion planning and control algorithms. However, even for identical multibody systems, there are distinct motion representations in the literature. These formulations differ both in terms of their algebraic form and some key properties. The goal of this paper is to present a common derivation for the forward dynamics model of a constrained system, based on known analytical dynamics' principles [32], and relate this result with the two

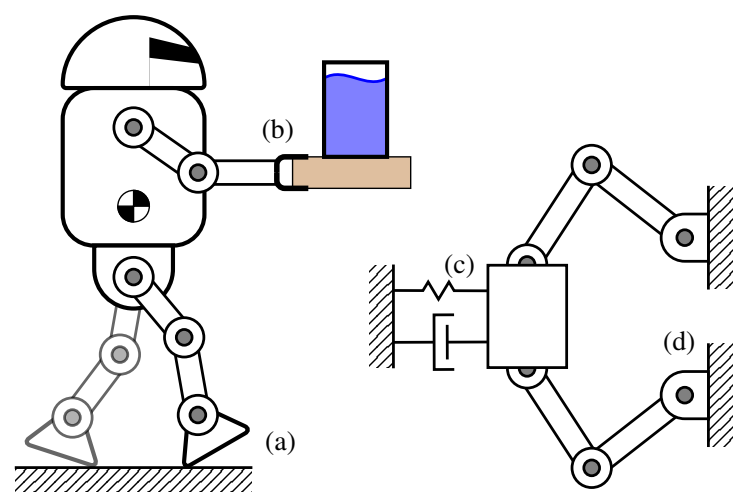

Fig. 1. Illustration of different constraints imposed by the robot's surroundings or required behaviour. Examples are: (a) using contacts for bipedal locomotion; (b) keeping the balance while executing a critical task such as holding a jar of water; (c) having a compliant behaviour while following a given trajectory; (d) and robots with closed kinematic loops.

main approaches found in the robotics literature.

Based on these two motion representations, various authors propose numerous control structures designed to achieve particular desired behaviours, by optimizing different criteria. Khatib [20] proposes the Operational Space Formulation (OSF) as a methodology for the description of the endeffector/tool constrained motion task. This work relies on the definition of a dynamically consistent inverse Jacobian, as a way of controlling redundant robots without affecting the specified end-effector task motion. Numerous studies follow and extend this formulation. For instance: Sentis and Khatib $[29,30]$ propose a task prioritization framework for control of humanoid robots, achieving complex behaviours by the activation or deactivation of different tasks and constraints, and their ordering in the pool of control primitives; Park and Khatib [26] address multiple contacts and the transition between those contacts in the control of humanoid robots; Nakanishi et al. [24] compare it with velocity and acceleration based controllers, concluding that this formulation is quite sensitive to modeling errors when compared to the other kinematic-based approaches; and De Sapio and Khatib [9] incorporate time independent equality constraints (scleronomic constraints) into the operational space formulation, highlighting the symmetry between constraints and tasks.

Aghili [1, 2] proposes a Projection-based Dynamics (PbD) approach for the derivation of the rigid multi-body dynamic equations of motion, subject to scleronomic equality con- 


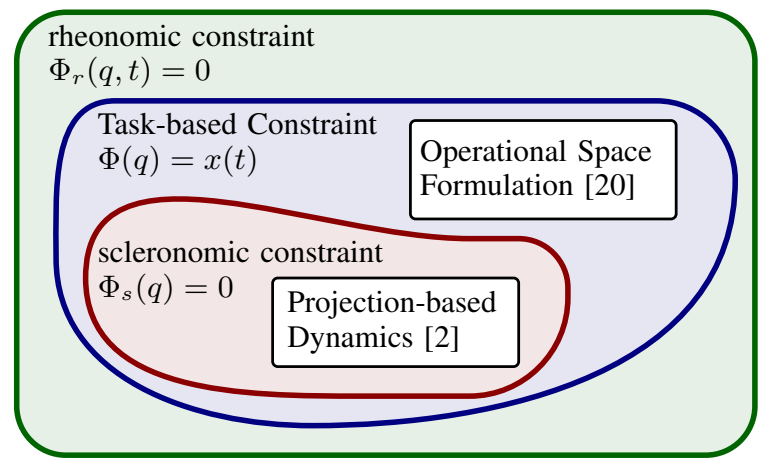

Fig. 2. Diagram illustrating the categorization of the two forward dynamic approaches discussed in this paper, regarding their underlying equality holonomic constraint. A rheonomic constraing is a time dependent constraint, a scleronomic constraint is a time independent constraint, and a Task-based Constraint is a time dependent constraint with decoupled dependence on the configuration $q$ and time $t$. Note that despite the $\mathrm{PbD}$ being mostly applied to scleronomic constraints, in an earlier work Aghili and Piedbœuf [4] demonstrate its application to rheonomic constraint. The categorization in this diagram corresponds to the context of explicitly considering a task component in the constraint formulation.

straints. This work relies on the definition of a constraint inertia matrix, in order to represent the constrained forward dynamics in the configuration space. Numerous studies follow and extend this approach. For instance: Mistry and Righetti [23] derive operational space controllers for constrained systems with passive joints; Ortenzi et al. [25] integrate the Projected Inverse Dynamics in an optimal control framework for robots in contact; Lin et al. [22] propose a control framework for multi-arm Cartesian impedance control; Dehio et al. [11] model and control multi-arm and multi-legged robots, while compensating for object dynamics, enabling humanrobot interaction.

When contrasting these two main approaches, we need to analyse both their domain of application and key properties. Regarding the domain of application, the OSF is a framework for describing and controlling task space motions, whereas the $\mathrm{PbD}$ is an approach for describing and controlling constrained multi-body systems. De Sapio and Khatib [9] extend the OSF to include constrained systems, and Mistry and Righetti [23] extend the PbD to include task motions, in which case the domain of application is the same. Regarding their key properties: the OSF requires a full rank robot Jacobian while the $\mathrm{PbD}$ handles a rank deficient constraint Jacobian; and the OSF uses an oblique projection (based on the dynamically consistent inverse) whereas the $\mathrm{PbD}$ relies on an orthogonal projection.

Despite these differences, we show that these approaches are equivalent. Our work takes inspiration from the more general treatment of equality constraints from Udwadia and Kalaba [32], and the parallels between task space and constraint formulations drawn by De Sapio et al. [10]. We start by defining a class of constraints called Task-based Constraint $(\mathrm{TbC})$, with the aim of unifying both OSF and $\mathrm{PbD}$ in terms of their domain of application. This class of constraints is in itself a sub-class of a general type of holonomic equality time dependent constraint (rheonomic constraints). Fig. 2 illustrates the relationship between the different types of equality constraints mentioned, and their relation to OSF and $\mathrm{PbD}$.

An immediate repercussion of proving the equivalence between OSF and $\mathrm{PbD}$ is that we can use either for computing the forward dynamics of a constrained system, with both leading to the same dynamically consistent result. From this point of view, regarding the simulation of a multi-body system, the only reason for choosing one method over the other is seeking some numerical advantage, as reduced numerical errors or reduced speed of computation. There are studies that demonstrate efficient computation of the dynamically consistent inverse Jacobian, as [33] for a $6 \times n$ end-effector Jacobian, and [17] for branched kinematic trees, that might grant the OSF some computational speed gains for these particular cases. On the other hand, given the flexibility of $\mathrm{PbD}$ approach in choosing different algebraic expressions for the constraint inertia matrix, theoretically we could find a matrix that might grant us some ease in its inversion, both from a computational speed and numerical errors perspective. However, so far we have found no evidence of the numerical superiority of either method for a general case. There are also some works on the stability analysis [17] and asymptotic stability for the regulation case of a passivity-based OSF [12], that should readily apply to $\mathrm{PbD}$.

The contributions of this paper are:

- We prove the equivalence between the OSF and PbD, regarding the forward constrained dynamics:

- We show that OSF generalizes to a rank deficient Jacobian, and prove that its solution is still dynamically consistent;

- This generalization allow us to numerically handle linearly dependent constraints, without requiring the use of other methods, such as SVD, to find a reduced number of independent constraints.

- We reformulate the PbD constraint inertia matrix, such that it generalizes the multiple algebraic solutions proposed by Aghili [2].

- This reformulation provides us with a mechanism of generating other algebraic expressions for the constraint inertia matrix.

- We present a multiple TbC abstraction that generalizes the operational space equations of motion with constraints.

- We show that the operational space equations of motion with constraints independently developed by De Sapio and Khatib [9] and Mistry and Righetti [23] are also equivalent.

\section{RELATED WORK AND PRELIMINARIES}

We first define a generic kinematic constraints. We then extend this formulation to a general forward constrained dynamical solution which we use to derive our proof.

\section{A. Task-based Constraint Kinematics}

Let $q \in \mathcal{D}_{q} \subset \mathbb{R}^{n}$ be the vector of generalized coordinates or configuration of a rigid multi-body system. We define a 
Task-based Constraint as any constraint written in the form

$$
\Phi(q)=x(t)
$$

where $x \in \mathcal{D}_{x} \subset \mathbb{R}^{m}$ is the vector of the task space coordinates $^{1}$, with $m \leq n$, and $t$ represents time. The function $\Phi: \mathcal{D}_{q} \mapsto \mathcal{D}_{x}$ - that maps the configuration space to task space - is, in general, a non linear function that captures the geometric model of the given constraint.

From a robotics perspective, the task $x$ might be: nonexistent, i.e. $x=0$, corresponding to some rigid link connection; externally enforced but still time varying, which might be through connection to a moving rail or caused by another agent (human or robotic); or enforced by the robot itself by an appropriate task controller. Regardless of task enforcing mechanism, it's effect is always the reduction of the configuration space domain $q \in \mathcal{D}_{q}=\left\{q \in \mathbb{R}^{n}, x \in \mathcal{D}_{x} \mid \Phi(q)=x\right\}$.

Differentiating Eq. (1) leads to

$$
A(q) \dot{q}=\dot{x}(t)
$$

where the matrix $A=(\delta \Phi / \delta q) \in \mathbb{R}^{m \times n}$ corresponds to the constraint Jacobian, $\dot{q} \in \mathcal{Q} \subset \mathbb{R}^{n}$ is the generalized velocity, and $\dot{x} \in \mathbb{R}^{m}$ is the task velocity. The second time derivative of Eq. (1) becomes

$$
A(q) \ddot{q}=\ddot{x}(t)-\dot{A}(q, \dot{q}) \dot{q},
$$

where $\ddot{q} \in \mathcal{A} \subset \mathbb{R}^{n}$ is the generalized acceleration, and $\ddot{x} \in \mathbb{R}^{m}$ is the task acceleration. We will use the differential constraint for formulating the inverse kinematics and forward dynamic equations.

\section{B. Task-based Constraint Inverse Kinematics}

If we have direct control authority over the configuration velocity $\dot{q}$ and wish to track a desired task trajectory/path $x(t)$ and $\dot{x}(t)$, we can solve Eq. (2) for $\dot{q}$. However, for a redundant robotic system $(m<n)$, there is an infinite number of solutions. The general solution is

$$
\dot{q}=G \dot{x}+\left(I_{n}-G A\right) \dot{q}_{\varepsilon},
$$

where $G$ is any generalized inverse (or G-inverse) of $A, \dot{q}_{\varepsilon} \in$ $\mathbb{R}^{n}$ is an arbitrary configuration velocity, and $I_{n}$ is $n \times n$ identity matrix. A G-inverse of $A$ is a matrix that satisfies the condition $A G A=A[6,32]$.

The most widely applied type of G-inverse in the robotics literature is the Moore-Penrose inverse (MP-inverse), often called pseudo-inverse [31, 14]. The MP-inverse of $A$ is the unique matrix $G=A^{\dagger}$ that satisfies the 4 conditions: (i) $A G A=A$ (ii) $G A G=G$ (iii) $A G=(A G)^{\top}$ (iv) $G A=(G A)^{\top}$, and it emerges from the solution $\dot{q}=A^{\dagger} \dot{x}$ to Eq. (2) of minimum-norm $\|\dot{q}\|^{2}=\langle q, q\rangle$ that minimizes the least-square error $\|A \dot{q}-\dot{x}\|^{2}[13,32]$, where $\langle$,$\rangle repre-$ sents an Euclidean inner product. For full row rank $A$, the MP-inverse assumes the widely used closed form solution $A^{\dagger}=A^{\top}\left(A A^{\top}\right)^{-1}$, known as right inverse.

\footnotetext{
${ }^{1}$ Here we dropped the time dependency on $x$. For readability purposes, we shall drop input dependencies after introducing dependent variables.
}

Another widely used unique G-inverse is the inertiaweighted generalized inverse $G=\bar{A}$ [10], that arises from the solution $\dot{q}=\bar{A} \dot{x}$ to Eq. (2) that minimizes the instantaneous kinetic energy $\frac{1}{2}\|\dot{q}\|_{M}^{2}=\frac{1}{2}\langle\dot{q}, \dot{q}\rangle_{M}=\frac{1}{2}\langle\dot{q}, M \dot{q}\rangle$ of a multibody system, with inertia matrix $M(q)$, while minimizing the least-square error $\|A \dot{q}-\dot{x}\|^{2}$. This G-inverse satisfies the first $3 \mathrm{MP}$-inverse conditions, with the additional condition $M G A=(M G A)^{\top}$ [6]. For full row rank $A$ we get the closed form solution $\bar{A}=M^{-1} A^{\top}\left(A M^{-1} A^{\top}\right)^{-1}$ [20].

The second term of the sum in Eq. (4) is a projection matrix $\left(I_{n}-G A\right)$, that projects any arbitrary configuration velocity $\dot{q}_{\varepsilon}$ to the null space of $A$, i.e. $\left(I_{n}-G A\right) \dot{q}_{\varepsilon} \in \mathcal{N}(A)$. This projection operator can be defined for both $\bar{A}$, where $\bar{P} \triangleq$ $\left(I_{n}-\bar{A} A\right)$ is an oblique inertia-weighted projection matrix [10], and for $A^{\dagger}$, where $P \triangleq\left(I_{n}-A^{\dagger} A\right)$ is an orthogonal projection, i.e. $P=P^{\top}$.

This constraint formulation can be further extended to a dynamical system by combining it with the dynamics of the unconstrained dynamical system.

\section{Configuration Space Dynamics}

The equation of motion of an unconstrained system in the configuration space is

$$
M\left(q_{\star}\right) \ddot{q}_{\star}+h\left(q_{\star}, \dot{q}_{\star}\right)=\tau
$$

where $h \in \mathbb{R}^{n}$ contains the Coriolis, centrifugal, and gravitational contributions, $M\left(q_{\star}\right)$ is the unconstrained inertia matrix, $\tau \in \mathbb{R}^{n}$ is the generalized force vector in the configuration space, and $q_{\star}, \dot{q}_{\star}, \ddot{q}_{\star} \in \mathbb{R}^{n}$ are, respectively, the unconstrained generalized position, velocity, and acceleration. For an unconstrained system where $M$ is a symmetric positive definite, we can compute the forward dynamics by simply inverting $M$ as

$$
\ddot{q}_{\star}=M^{-1}(\tau-h) .
$$

There are of course more efficient methods for computing $\ddot{q}_{\star}$ [16], we shall use this expression just for constrained system derivation purposes.

\section{Task Space Dynamics}

One way of taking into account the contribution of a Taskbased Constraint in the dynamics equation of motion of a multi-body system is to simply add that extra force component to the equation of motion as,

$$
M \ddot{q}+h-A^{\top} \lambda=\tau,
$$

where $\lambda \in \mathbb{R}^{m}$ is the force coming from the task space.

We obtain the task space dynamics equation of motion by applying the operational space formulation from [20], resulting in

$$
M_{x} \ddot{x}+h_{x}-\lambda=f,
$$

where

$$
M_{x} \triangleq\left(A M^{-1} A^{\top}\right)^{-1}=\bar{A}^{\top} M \bar{A}
$$

is the task space inertia matrix [18], and with $h_{x} \triangleq \bar{A}^{\top} h-$ $M_{x} \dot{A} \dot{q}$, and $f \triangleq \bar{A}^{\top} \tau$, for $A$ full row rank. 


\section{E. Projected Dynamics}

Aghili $[1,2]$ defines the Projected Inverse Dynamics of a multibody system by pre-multiplying Eq. (7) with the orthogonal projector $P$, obtaining

$$
P M \ddot{q}=P(\tau-h) .
$$

For computing the forward dynamics equation of motion, as $P M$ is singular, we pre-multiply Eq. (3) with $A^{\dagger}$, obtaining

$$
\left(I_{n}-P\right) \ddot{q}=A^{\dagger}(\ddot{x}-\dot{A} \dot{q}),
$$

and combine it with Eq. (10), obtaining

$$
M_{c} \ddot{q}=P(\tau-h)+C_{c}(\ddot{x}-\dot{A} \dot{q})
$$

where $M_{c}$ is called the constraint inertia matrix. As $M_{c}$ is invertible, we get

$$
\ddot{q}=M_{c}^{-1} P(\tau-h)+M_{c}^{-1} C_{c}(\ddot{x}-\dot{A} \dot{q}),
$$

which Aghili [2] calls equation of motion of a constrained system in a compact form. ${ }^{2}$

Depending on different ways of combining Eq. (10) and Eq. (11), both $M_{c}$ and $C_{c}$ take different expressions. Aghili $[1,2]$ derives and discusses the numerical properties of the following different combinations of $M_{c}$ and $C_{c}$ :

1) $M_{c}^{(1)}=P M+\left(I_{n}-P\right), C_{c}^{(1)}=-A^{\dagger}$,

2) $M_{c}^{(2)}=M+P M+(P M)^{\top}, C_{c}^{(2)}=-M A^{\dagger}$,

3) $M_{c}^{(3)}=P M P+\left(I_{n}-P\right) M\left(I_{n}-P\right)$, $C_{c}^{(3)}=-\left(I_{n}-2 P\right) M A^{\dagger}$,

4) $M_{c}^{(4)}=P M+\gamma\left(I_{n}-P\right), C_{c}^{(4)}=-\gamma A^{\dagger}$,

where $\gamma$ is a non-negative scalar.

\section{F. General Forward Constrained Dynamics Solution}

Analogously to what was done for the general solution of an constrained inverse kinematics problem, the general solution for the Task-based Constrained configuration acceleration from Eq. (3) is

$$
\ddot{q}=G(\ddot{x}-\dot{A} \dot{q})+\left(I_{n}-G A\right) \ddot{q}_{\varepsilon},
$$

with $\ddot{q}_{\varepsilon} \in \mathbb{R}^{n}$ being an arbitrary configuration acceleration. We can then argue that any forward dynamics solution, i.e. expression for computing a $\ddot{q}$, is a particular case of the general solution (14), corresponding to particular choices of $G$ and $\ddot{q}_{\varepsilon}$, and resulting from optimizing different cost functions. For instance: $\ddot{q}=A^{\dagger}(\ddot{x}-\dot{A} \dot{q})$ minimizes $\|\ddot{q}\|^{2}$, being a particular case of Eq. (14) for which $G=A^{\dagger}$ and $\ddot{q}_{\varepsilon}=0 ; \ddot{q}=\bar{A}(\ddot{x}-\dot{A} \dot{q})$ minimizes $\frac{1}{2}\|\dot{q}\|_{M}^{2}$ and corresponds to the case where $G=\bar{A}$ and $\ddot{q}_{\varepsilon}=0$.

\footnotetext{
${ }^{2}$ Note that here we followed the same derivation process as in [1] but adding as task acceleration $\ddot{x}$. By making $\ddot{x}=0$, we recover the original results. Therefore, to accommodate $\ddot{x}$ we removed $\dot{A}$ from $C_{c}$.
}

\section{G. Forward Constraint Dynamics: Gauss' Principle}

One can obtain another particular case of Eq. (14), by using a fundamental principle of mechanics - the Gauss' Principle of Least Constraint - which states that if a given configuration acceleration $\ddot{q}$ simultaneously satisfies the constraint and minimizes the Gauss function

$$
\mathcal{G}(\ddot{q})=\left\langle\ddot{q}_{\star}-\ddot{q}, \ddot{q}_{\star}-\ddot{q}\right\rangle_{M},
$$

where $\ddot{q}_{\star}$ is the unconstrained acceleration, then $\ddot{q}$ is the correct acceleration the constrained system will acquire, i.e. that is the acceleration that actually materializes $[32,7,10]$. The result of that minimization is

$$
\begin{aligned}
\ddot{q} & =\bar{A}(\ddot{x}-\dot{A} \dot{q})+\bar{P} \ddot{q}_{\star} \\
& =\bar{A}(\ddot{x}-\dot{A} \dot{q})+\bar{P} M^{-1}(\tau-h),
\end{aligned}
$$

where we can see that is a particular solution of Eq. (14) for which $G=\bar{A}$ and $\ddot{q}_{\varepsilon}=\ddot{q}_{\star}$.

Udwadia and Kalaba [32] derives the Fundamental Equation

$$
\ddot{q}=\ddot{q}_{\star}+\bar{A}\left(b-A \ddot{q}_{\star}\right),
$$

which is a equivalent way of writing Eq. (16), but covers a much more general class of non-holonomic equality constraint written in the Pfaffian form $A(q, \dot{q}, t) \ddot{q}=b(q, \dot{q}, t)$. Therefore, Eq. (16) is a sub-case of the Fundamental Equation for which $b=\ddot{x}-\dot{A} \dot{q}$. Furthermore, Udwadia and Kalaba [32] consider the case of a rank deficient $A$, by using the following inertiaweighted generalized inverse, ${ }^{3}$

$$
\bar{A}=M^{-1} A^{\top}\left(A M^{-1} A^{\top}\right)^{\dagger} .
$$

In the following sections we will relate this remarkably simple result with the constraint forward dynamic solutions from the $\mathrm{OSF}$ and $\mathrm{PbD}$.

\section{Operational/Task Space Formulation GENERALIZATION}

\section{A. The Dynamically Consistent Generalized Inverse}

The same way that in Eq. (4) we decompose $\dot{q}$ into a task component and null-space component using a G-inverse, Khatib [20] decomposes $\tau$ in its task and null-space components using $A^{\top}$ as

$$
\tau=A^{\top} f+\left(I_{n}-A^{\top} G^{\top}\right) \tau_{\varepsilon}
$$

where $\tau_{\varepsilon} \in \mathbb{R}^{n}$ is an arbitrary generalized force vector.

Khatib [21] defines the Dynamically Consistent Inverse of a robot Jacobian $A$ as the matrix $G$ that satisfies the condition

$$
A M^{-1}\left(I_{n}-A^{\top} G^{\top}\right) \tau_{\varepsilon}=0,
$$

which corresponds to the solution that decouples the generalized force $\tau$ into a component $A^{\top} f$ only acting in the robot's end-effector, and a component $\left(I_{n}-A^{\top} G^{\top}\right) \tau_{\varepsilon}$ that

\footnotetext{
${ }^{3}$ Udwadia and Kalaba [32] discuss other expressions for the inertiaweighted generalize inverse that still comply with the Gauss' Principle of Least Constraint.
} 
only affects the internal motion of the robot, i.e. the task space motion is unaffected by $\tau_{\varepsilon}$. Furthermore, Khatib [21] proves that for full rank $A$, the only G-inverse that satisfies such condition is the inertia-weighted generalized inverse [18].

The first straightforward generalization of the original OSF is its application to any Task-based Constraint by replacing the end-effector robot Jacobian by any constraint Jacobian $A$, that splits the space of motion into a task space of interest and it's null space. The second generalization is to extend it to the case where $A$ is rank deficient, which allow us to numerically handle redundant constraints and singular configurations. For that we simply use the inertia-weighted generalized inverse of Eq. (18) and redefine the task space inertia matrix from Eq. (9) as

$$
M_{x} \triangleq\left(A M^{-1} A^{\top}\right)^{\dagger}=\bar{A}^{\top} M \bar{A} .
$$

Note that for $A$ full rank, then Eq. (21) is equivalent to Eq. (9). Furthermore, we can prove that any inertia-weighted generalized inverse is still dynamically consistent, one important principle in the OSF to guarantee that task space controllers remain unaffected by null space controllers.

Lemma 1: Let $G=\bar{A}$ be the unique inertia-weighted generalized inverse of a rank deficient Jacobian $A$, then this generalized inverse satisfies the condition from Eq. (20) being, therefore, a dynamically consistent inverse of the Jacobian $A$. The proof of this lemma can be found in Appendix A.

\section{B. Equivalence with the Least Constraint Solution}

Peters et al. [27] and Bruyninckx and Khatib [7] already discuss the equivalence of the operational space control expressions with the results obtained using the Gauss' Principle of Least Constraint. We can easily show that by rearranging Eq. (8) with respect to the constraint force $\lambda$ and substituting it in Eq. (7) and then inverting the inertia matrix $M$, we obtain the same solution of Eq. (16). Furthermore, by generalizing the Operational Space equations of motion to $A$ rank deficient, then this equivalence is also valid for any $A$.

\section{Projected Dynamics Reformulation}

We reformulate the constraint inertia matrix $M_{c}$ as

$$
M_{c} \triangleq P M+R\left(I_{n}-P\right),
$$

and $C_{c}$ as

$$
C_{c} \triangleq-R A^{\dagger} .
$$

It is straight forward to show, through simple algebraic manipulations, that all choices of $M_{c}$ and $C_{c}$ presented by Aghili [2] are particular instances of Eq. (22) and Eq. (23), where the matrix $R$ respectively takes the following expressions: 1) $R^{(1)}=I_{n}$, 2) $R^{(2)}=M$, 3) $R^{(3)}=\left(I_{n}-2 P\right) M$, 4) $R^{(4)}=\gamma I_{n}$. We can then re-write Eq. (13) as

$$
\ddot{q}=M_{c}^{-1} P(\tau-h)+M_{c}^{-1} R A^{\dagger}(\ddot{x}-\dot{A} \dot{q}) .
$$

In fact, we will show later that $R$ can be any square matrix such that $M_{c}$ is full rank. We can even use a matrix $R^{(r)}$ whose elements are randomly generated, as long as we check the rank of $M_{c}$, and the solution of Eq. (24) will remain the same. This happens, because the combination of Eq. (10) and Eq. (11) sole purpose is to invert the projected inverse dynamics (10) and, therefore, we have to add some component to Eq. (10) in order to make $P M$ full rank. We can even look at this solution as a special type of regularization, where the regularization term $R$ only affects the complement space of the motion of interest.

Two important benefits of this reformulation are: firstly, any proof done for these generalized $M_{c}$ and $C_{c}$ is directly valid for all the other ones presented in the literature; and secondly, we have now a mechanism of obtaining new $M_{c}$ and $C_{c}$ based on finding $R$ such that $M_{c}$ and $C_{c}$ satisfy some desired property. For example, we can find $R$ that leads to the $M_{c}$ with the smallest condition number among all possible constrained inertia matrices, which is a desirable numerical property [2].

Lemma 2: The $R^{(*)}$ that minimizes $\kappa\left(M_{c}\right)$, where $\kappa($. represents the condition number, is given by

$$
R^{(*)}=\mu I_{n}-P M,
$$

yielding $M_{c}^{(*)}=P M P+\left(\mu I_{n}-P\right)$, for some $\mu \in \mathbb{R}$ such that $\left\{\sigma_{\min }(P M P) \neq 0\right\} \leq \mu \leq \sigma_{\max }(P M P)$, where $\sigma($. represents singular values. Furthermore, $\kappa\left(M_{c}^{(*)}\right)$ is equivalent to $\kappa\left(Z_{2}^{\top} M Z_{2}\right)$, where $Z_{2}$ is a basis for $\mathcal{N}(A)$ such that $P=$ $Z_{2} Z_{2}^{\top}$.

The proof of this lemma can be found in Appendix B.

\section{A. Case Study for the Inertia Matrix's Condition Number}

Aghili [2] provides a detailed analysis on the symmetry and positive definiteness properties of each $M_{c}$ proposed, key properties for its inversion. Another key property to consider for the inversion of $M_{c}$ is its condition number. For illustration purposes, we simulated a free fall motion of a three link planar serial robot arm with its end-effector constrained to a vertical slider, as shown in Fig. 3. We then computed the condition number for $M$ and for the different $M_{c}$ 's discussed in this paper, as shown in Fig. 4.

The robot arm used consists of a planar serial robot arm (Fig. 3a), composed of three identical links with equidistant center of mass from the joints, and with length, mass, and inertia of $1 \mathrm{~m}, 1 \mathrm{~kg}$, and $0.1 \mathrm{~m} \mathrm{~kg}^{2}$, respectively. We used Corke [8]'s MatLab® toolbox for computing $M, h, \ddot{q}_{\star}$, and the robot's Jacobian. For the forward simulation of the motion we integrated Eq. (24) using a non-adaptive (fixed step) DormandPrince solver of order 5, obtaining 200 samples corresponding to $2 \mathrm{~s}$. We set $\tau=0$ and $\ddot{x}=0$, meaning the robot is nonactuated (free fall) and there is no task space (scleronomic constrait). Fig. $3 b$ shows the resulting joint angles.

In Fig. 4 we can verify, as expected, that the condition number curve corresponding to $M_{c}^{(*)}$ is a lower bound for all other condition number curves. However, for this illustrative case study $M$ is too well conditioned, i.e. $\kappa(M) \ll$ roundoff error, to expect any significant impact in the numerical errors resulting from using different $M_{c}$ 's. For example, if we compute the joint accelerations for the trajectory in Fig. $3 b$ using any two of the $M_{c}$ 's discussed, the mean norm of their 


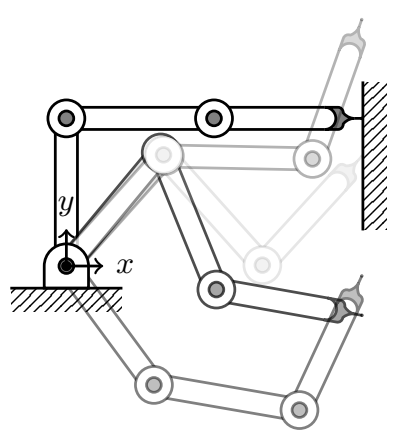

(a)

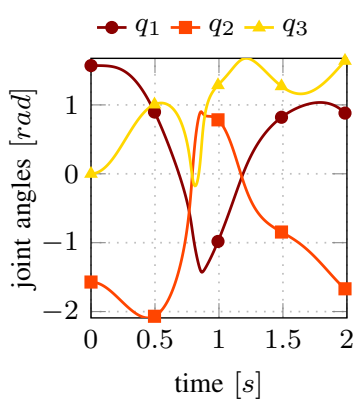

(b)
Fig. 3. Free fall (i.e. $\tau=0$ ) simulation of a two dimensional serial robot arm with three links and with the end-effector constrained to a vertical slider: (a) five samples of configurations taken during the free fall motion, with less opaque configurations corresponding to further in the time of the simulation (b) time evolution of the arm joint angles, with $q_{1}$ being the angle of the base joint and $q_{3}$ the angle of the last joint, and with markers placed at time instances corresponding to the samples in (a).

difference is in the order of magnitude of the MatLab's roundoff error $\left(10^{-14}\right)$.

One could hypothesize that, for a badly conditioned $M$, using the method that requires a matrix inversion with lower condition number would result in smaller simulation errors, specially when using $M_{c}^{(*)}$. However, in all our experiments (other simulations involved serial arms with non-identical and larger number of links) this hypothesis failed to hold, as we always obtained the same constraint error propagation regardless of the $M_{c}$ employed or even when using Eq. (16).

Featherstone [15] discusses that the ill-conditioning of $M$ is more than a numerical artifact, but a phenomenon of the underlying mechanism of the multi-body system itself. We might reason that for systems and configurations where $\kappa(M)$ is large, i.e. $\kappa(M) \approx$ round-off error, the minimum condition number $\kappa\left(Z_{2}^{\top} M Z_{2}\right)$ will also approach a large value due to its dependence on $M$. Even though some of our experiments confirm our reasoning, until proved or extensively tested, it will remain an open question. Note that we found slight variations of the constraint error and simulation time when using different variable step integration solvers, that somehow prefer some $M_{c}$ structures over the others, but without identifying a distinct pattern.

\section{B. Equivalence with the Least Constraint Solution}

As noted in the previous case study, in all our experiments we verified that using either the Principle of Least Constraint or any of the $\mathrm{PbD}$ solutions always resulted in the same constrained acceleration. Equipped with our reformulated $\mathrm{PbD}$ Eq. (24) parameterized by $R$, we can now easily compare the Least Constraint solution, given by Eq. (16), with the PbD. We hypothesize that they are analytically equivalent, which by inspection of expressions (16) and (24) leads to the following Lemma.

Lemma 3: For any $R \in \mathbb{R}^{n \times n}$ such that $M_{c}$ is invertible,

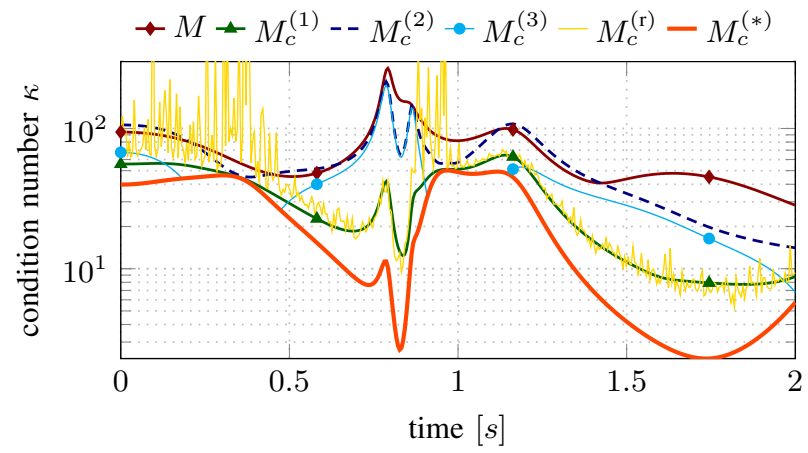

Fig. 4. Time evolution of the condition number for different constrained inertia matrices and for the unconstrained inertia matrix. We compute $M_{c}^{(*)}$ using $R^{(*)}$ from Eq. (25), and $M_{c}^{(r)}$ using a different $R^{(r)}$ for every time iteration and with elements sampled from an uniform distribution in the interval $[0,1]$.

we have that

$$
\begin{aligned}
\bar{A} & =M_{c}^{-1} R A^{\dagger}, \\
\bar{P} M^{-1} & =M_{c}^{-1} P
\end{aligned}
$$

The proof of this lemma can be found in Appendix C.

\section{Multiple Constraints}

So far we treated $A \in \mathbb{R}^{m \times n}$ as a single constraint, but in reality it accommodates multiple constraints acting simultaneously. In this section we consider the case where we partition $A$ into two and treat it as two separate constraints acting simultaneously. This treatment readily generalizes to any other number of partitions.

If we split the task space vector $\dot{x}$ into two components $\dot{x}_{1}$ and $\dot{x}_{2}$, we can think each of these tasks as being associated by a different constraint $A_{1}$ and $A_{2}$, respectively, such that the constraint Jacobian is $A=\left[\begin{array}{ll}A_{1}^{\top} & A_{2}^{\top}\end{array}\right]^{\top}$. Therefore, the task space inertia matrix becomes [34]

$$
\begin{aligned}
M_{x} & =\left[\begin{array}{cc}
A_{1} M^{-1} A_{1}^{\top} & A_{1} M^{-1} A_{2}^{\top} \\
A_{2} M^{-1} A_{1}^{\top} & A_{2} M^{-1} A_{2}^{\top}
\end{array}\right]^{\dagger} \\
& =\left[\begin{array}{cc}
M_{1} & -\bar{A}_{1}^{\top} A_{2}^{\top} M_{2} \\
-\bar{A}_{2}^{\top} A_{1}^{\top} M_{1} & M_{2}
\end{array}\right],
\end{aligned}
$$

where

$$
M_{1} \triangleq\left(A_{1} \bar{P}_{2} M^{-1} A_{1}^{\top}\right)^{\dagger} \quad \text { and } \quad M_{2} \triangleq\left(A_{2} \bar{P}_{1} M^{-1} A_{2}^{\top}\right)^{\dagger}
$$

with $\overline{A_{1}}$ and $\overline{A_{2}}$ being, respectively, the dynamically consistent inverse of $A_{1}$ and $A_{2} . \bar{P}_{1}$ and $\bar{P}_{2}$ are the respective projection matrices. The dynamically consistent inverse of the two constraints combined is given by

$$
\bar{A}^{\top}=\left[\begin{array}{l}
\left(A_{1} \bar{P}_{2} M^{-1} A_{1}^{\top}\right)^{\dagger} A_{1} \bar{P}_{2} M^{-1} \\
\left(A_{2} \bar{P}_{1} M^{-1} A_{2}^{\top}\right)^{\dagger} A_{2} \bar{P}_{1} M^{-1}
\end{array}\right] \triangleq\left[\begin{array}{c}
A_{1}^{\#^{\top}} \\
A_{2}^{\#}
\end{array}\right]
$$

where we define $A_{1}^{\#^{\top}}$ and $A_{2}^{\#^{\top}}$ to be the partial dynamically consistent inverses. By partitioning the task force $f=$ $\left[\begin{array}{ll}f_{1}^{\top} & f_{2}^{\top}\end{array}\right]^{\top}$ we can then obtain the separate sub task dynamic 
equations, potentially allowing us to apply different control schemes to each task space. For example, we have

$$
f_{2}=M_{2}\left(\ddot{x}_{2}-\dot{A}_{2} \dot{q}\right)-\bar{A}_{2} A_{1}^{\top} M_{1}\left(\ddot{x}_{1}-\dot{A}_{1} \dot{q}\right)+A_{2}^{\#}{ }^{\top} h-\lambda_{2}
$$

for the task 2, and the force of the task 1 takes an analogous expression.

\section{A. Operational Space Control with Constraints}

As we showed, the forward projected dynamics is equivalent to the dynamically consistent solution, therefore, we must also draw an equivalence between different methods for operational space control with constraints.

If we call $A_{1}$ the Jacobian of the constraint and $A_{2}$ the Jacobian of the robot, and set $\ddot{x}_{1}=0$, then the partition in (28) coincides exactly with the partition from [9]. Therefore, for $\lambda_{2}=0$ and $\ddot{x}_{1}=0$, Eq. (30) corresponds to the task motion control, and its homologous for $f_{1}$ corresponds to the constraint forces.

Regarding the approach based on the projected inverse dynamics, knowing that $\bar{P} M^{-1}=M_{c}^{-1} P$, we see that the partial dynamically consistent inverse takes the form

$$
\begin{aligned}
A_{2}^{\#^{\top}} & \triangleq\left(A_{2} \bar{P}_{1} M^{-1} A_{2}^{\top}\right)^{-1} A_{2} \bar{P}_{1} M^{-1} \\
& =\left(A_{2} M_{c}^{-1} P_{1} A_{2}^{\top}\right)^{-1} A_{2} M_{c}^{-1} P_{1},
\end{aligned}
$$

which is the same used in [23]. Furthermore, by performing some algebraic operations on Eq. (30) and by making $R=I_{n}$, $\lambda_{2}=0$, and $\ddot{x}_{1}=0$, we recover

$$
f_{2}=M_{2}\left[\ddot{x}_{2}+A_{2} M_{c}^{-1} P_{1} h-\left(\dot{A}_{2}-A_{2} M_{c}^{-1} R A_{1}^{\dagger} \dot{A}_{1}\right) \dot{q}\right]
$$

which is again exactly the same as the operational space dynamics presented with constraints found in [23]. This result shows that a framework of multiple Task-based Constraints generalizes the task space plus constraint formulations from De Sapio and Khatib [9] and Mistry and Righetti [23] and, furthermore, allow us to reason about them as equivalent.

\section{SUMMARY AND DISCUSSION}

In this paper, we define a class of Task-based Constraints (TbC) and derive the equations of motion of a multi-body system subject to that type of constraint. By contrasting our solution with the ones from the Operational Space Formulation (OSF) and Projection-based Dynamics $(\mathrm{PbD})$, we prove that those approaches are equivalent regarding the computation of the forward dynamics of a constrained multi-body system. In order to enable such equivalence, we generalize the OSF to a rank deficient Jacobian and reformulate the $\mathrm{PbD}$ to generalize all previous alternative algebraic expressions of the constraint inertia matrix. Moreover, we present a multiple $\mathrm{TbC}$ abstraction that generalizes the operational space equation of motion with constraints.

Some practical benefit of this Task-based Constraint abstraction is the convenience in expressing constraints using a task space formalization. For example, when Escande et al. [14] propose the Hierarchical Inverse Kinematics, applying it in the control of a humanoid robot, the task of reaching an object with the end-effector is in the null space of the task of keeping the balance of the humanoid. Similarly, Armesto et al. [5] propose a method for learning null space policies and applies it to learning a wiping motion, that when used to wipe a curved surface requires a task space controller for keeping contact with the surface. Sentis and Khatib [29, 30] also propose a task prioritization framework using dynamically consistent projections for the control of a humanoid, applying the different motion primitives in the null-space of the higher priority tasks. In all these works, the task motion with higher priority works as a constraint for the subsequent null space motion.

We now need to contrast our main finding with the one from Righetti et al. [28], who also discuss the equivalence between the OSF and the $\mathrm{PbD}$, but regarding the inverse dynamics computation (controller). They find that the output of the controller is independent of the projection operator used in that computation, assuming a desired constraint consistent configuration and a control signal that guarantees perfect tracking. Finally, they advocate for the use of an orthogonal projection (instead of an oblique) due to its computational simplicity, free from the inertia parameters. According to our finding, when computing that configuration acceleration (the forward dynamics) given a desired task motion, then both the OSF and the PbD approaches are also equivalent. However, in this case it is unclear which one is preferable, given that both of them use kinematic and inertia parameters, only with a different combination.

Going forward, finding what are the particular cases or numerical procedures that might give a significant advantage of one approach over the other, both in terms of the computational speed and numerical errors, can lead to some impactful practical benefits, resulting from our new theoretical understanding of Task-based Constrained multi-body dynamical systems.

\section{APPENDIX A}

Proof 1 (Lemma 1): Let $\bar{A}$ be the inertia-weighted generalized inverse of the rank deficient matrix $A$. Then $M \bar{A} A=$ $(M \bar{A} A)^{\top}$, which is equivalent to $\bar{A} A M^{-1}=\left(\bar{A} A M^{-1}\right)^{\top}[6]$, given $M$ symmetric positive definite. By replacing $G=\bar{A}$ in the condition from Eq. (20), we obtain

$$
\begin{aligned}
A M^{-1}\left(I_{n}-A^{\top} \bar{A}^{\top}\right) \tau_{\varepsilon} & =\left(A M^{-1}-A\left(\bar{A} A M^{-1}\right)^{\top}\right) \tau_{\varepsilon} \\
=\left(A M^{-1}-(A \bar{A} A) M^{-1}\right) \tau_{\varepsilon} & =\left(A M^{-1}-A M^{-1}\right) \tau_{\varepsilon}=0,
\end{aligned}
$$

for any $\tau_{\varepsilon} \in \mathbb{R}^{n}$, proving that $\bar{A}$ is a dynamically consistent inverse of the Jacobian matrix $A$.

\section{APPENDIX B}

Proof 2 (Lemma 2): The goal here is to find a matrix $R^{(*)}$ such that the condition number $\kappa$ of $M_{c}=P M+R\left(I_{n}-P\right)$ is minimal. By definition the condition number of a square matrix $C$ is

$$
\kappa(C) \triangleq\left\|C^{-1}\right\| \cdot\|C\|,
$$

for any consistent norm [19]. From definition (31), we can verify that the minimum possible condition number is 1 , that 
only happens if $C$ is a scalar multiple of a linear isometry, i.e. a distance preserving transformation. In the Euclidean space, such transformation is given by an orthogonal matrix $Q$. Therefore, if we can find $R$ such that $M_{c}=\mu Q$, where $\mu \in \mathbb{R}_{\neq 0}$, then that is the minimum possible condition number we can hope for. By equating $M_{c}$ and $\mu Q$, we obtain

$$
M_{c}=\mu Q \Leftrightarrow R\left(I_{n}-P\right)=\mu Q-P M .
$$

Given that $\left(I_{n}-P\right)$ is non invertible, it means the equality in Eq. (32) is false. However, if we post-multiply $\left(I_{n}-P\right)$ by both sides of Eq. (32), we get

$$
R\left(I_{n}-P\right)=(\mu Q-P M)\left(I_{n}-P\right),
$$

which is true for $R=(\mu Q-P M)$, and leads to the closest result of the approximation $M_{c} \approx \mu Q$. The resulting constraint inertia matrix is $M_{c}=\mu Q(I-P)+P M P$.

To keep generality, we can still consider $Q$ to be any square full rank matrix, thus all we have achieved so far is a rewritten $M_{c}$ in terms of $Q$ and $\mu$ instead of $R$. We shall now find a $Q$ and $\mu$ that minimize $\kappa\left(M_{c}\right)$. Given that $\left(I_{n}-P\right)$ is an orthogonal projection matrix, we can always find a partial isometry $Z_{1}$ such that $\left(I_{n}-P\right)=Z_{1} Z_{1}^{\top}$, and analogously $P=Z_{2} Z_{2}^{\top}$, where $Q_{p}=\left[\begin{array}{ll}Z_{1} & Z_{2}\end{array}\right]$ is an orthogonal matrix. We can then rewrite $M_{c}$ as

$$
\begin{aligned}
M_{c} & =\mu Q(I-P)+P M P=\mu Q Z_{1} Z_{1}^{\top}+Z_{2} Z_{2}^{\top} M Z_{2} Z_{2}^{\top} \\
& =\underbrace{\left[\begin{array}{ll}
Q Z_{1} & Z_{2}
\end{array}\right]}_{B} \underbrace{\left[\begin{array}{cc}
\mu I_{n-m} & 0 \\
0 & Z_{2}^{\top} M Z_{2}
\end{array}\right]}_{X} \underbrace{\left[\begin{array}{c}
Z_{1}^{\top} \\
Z_{2}^{\top}
\end{array}\right]}_{Q_{p}^{\top}} .
\end{aligned}
$$

We have that for any suitable norm

$$
\begin{aligned}
\left\|M_{c}\right\| & =\left\|B X Q_{p}^{\top}\right\|=\|B X\|, \\
\left\|M_{c}^{-1}\right\| & =\left\|Q_{p} X^{-1} B^{-1}\right\|=\left\|X^{-1} B^{-1}\right\|,
\end{aligned}
$$

and replacing the previous results in (31), we obtain

$$
\begin{aligned}
\kappa\left(M_{c}\right) & =\left\|M_{c}^{-1}\right\| \cdot\left\|M_{c}\right\|=\left\|X^{-1} B^{-1}\right\| \cdot\|B X\| \\
& \leq\left\|X^{-1}\right\| \cdot\|X\| \cdot\left\|B^{-1}\right\| \cdot\|B\| \\
& =\kappa(X) \kappa(B) .
\end{aligned}
$$

As $B$ only depends on $Q$ and $X$ only depends on $\mu$, we can independently find $Q$ and $\mu$ that minimize the respective $\kappa(B)$ and $\kappa(X)$. By inspection of Eq. (34) we see that $B$ is orthogonal if $Q=I_{n}$, in which case the inequality in Eq. (35) becomes an equality. Therefore, we obtain that the result that minimizes $\kappa\left(M_{c}\right)$ is $R^{(*)}=\left(\mu I_{n}-P M\right)$, for which

$$
M_{c}^{(*)}=\mu\left(I_{n}-P\right)+P M P .
$$

Aghili [3] more recently proposes an $M_{c}$ in the form of Eq. (36) and proves using a 2-norm that its condition number is minimum for $\left\{\sigma_{\min }(P M P) \neq 0\right\} \leq \mu \leq \sigma_{\max }(P M P)$, where $\sigma$ represents the singular values of a given matrix. We have that $\left\{\sigma_{\min }(P M P) \neq 0\right\}=\sigma_{\min }\left(Z_{2}^{\top} M Z_{2}\right)$ and $\sigma_{\max }(P M P)=\sigma_{\max }\left(Z_{2}^{\top} M Z_{2}\right)$. If we use a 2-norm in (31), then the condition number of a matrix is given by the ratio of its singular values. Therefore, by inspection of Eq. (34), we see that the minimium $\kappa\left(M_{c}^{(*)}\right)$ is $\kappa\left(Z_{2}^{\top} M Z_{2}\right)$.

\section{APPENDIX C}

Proof 3 (Lemma 3): The goal is to prove that $\bar{A}=$ $M_{c}^{-1} R A^{\dagger}$ and $\bar{P} M^{-1}=M_{c}^{-1} P$ for any $R \in \mathbb{R}^{n \times n}$ such that $M_{c} \in \mathbb{R}^{n \times n}$ is full rank. Using the MP-conditions that apply to $A^{\dagger}$ and the inertia-weighted generalized inverse conditions that apply to $\bar{A}$ (see Section II-B), we start by showing the intermediary results

$$
\begin{aligned}
P M \bar{A} & =M \bar{A}-A^{\dagger} A M \bar{A}=M \bar{A}-A^{\dagger} A M(\bar{A} A \bar{A}) \\
& =M \bar{A}-\left(A^{\dagger} A\right)^{\top}(M \bar{A} A)^{\top} \bar{A} \\
& =M \bar{A}-\left[\bar{A}^{\top} M \bar{A}\left(A A^{\dagger} A\right)\right]^{\top} \\
& =M \bar{A}-\left[\bar{A}^{\top}(M \bar{A} A)^{\top}\right]^{\top}=M \bar{A}-M(\bar{A} A \bar{A}) \\
& =M \bar{A}-M \bar{A}=0,
\end{aligned}
$$

and

$$
\begin{aligned}
\left(I_{n}-P\right) \bar{A} & =A^{\dagger} A \bar{A}=\left(A^{\dagger} A A^{\dagger}\right) A \bar{A}=A^{\dagger}\left(A A^{\dagger}\right)^{\top}(A \bar{A})^{\top} \\
& =A^{\dagger} A^{\dagger^{\top}}\left(A^{\top} \bar{A}^{\top} A^{\top}\right)=A^{\dagger} A^{\dagger^{\top}} A^{\top} \\
& =A^{\dagger}\left(A A^{\dagger}\right)^{\top}=A^{\dagger} A A^{\dagger}=A^{\dagger} .
\end{aligned}
$$

For $M_{c}$ invertible $\bar{A}=M_{c}^{-1} R A^{\dagger} \Leftrightarrow M_{c} \bar{A}=R A^{\dagger}$, and then we can show that

$$
\begin{aligned}
M_{c} \bar{A} & =\left[P M+R\left(I_{n}-P\right)\right] \bar{A}=P M \bar{A}+R\left(I_{n}-P\right) \bar{A} \\
& =R A^{\dagger} .
\end{aligned}
$$

Analogously, for $\bar{P} M^{-1}=M_{c}^{-1} P \Leftrightarrow M_{c} \bar{P} M^{-1}=P$, for which we can show that

$$
\begin{aligned}
M_{c} \bar{P} M^{-1} & =\left[P M+R\left(I_{n}-P\right)\right]\left[I_{n}-\bar{A} A\right] M^{-1} \\
& =\left[P M-P M \bar{A} A+R\left(I_{n}-P\right)\right. \\
& \left.-R\left(I_{n}-P\right) \bar{A} A\right] M^{-1} \\
& =\left[P M+R A^{\dagger} A-R A^{\dagger} A\right] M^{-1} \\
& =P M M^{-1}=P .
\end{aligned}
$$

\section{ACKNOWLEDGMENTS}

This work was supported by the European Union Horizon 2020, as part of the project Memory of Motion - MEMMO (project ID 780684), and the Engineering and Physical Sciences Research Council, UK, as part of the Robotics and AI hub in Future AI and Robotics for Space - FAIR-SPACE (grant number EP/R026092/1), and as part of the Centre for Doctoral Training in Robotics and Autonomous Systems at Heriot-Watt University and The University of Edinburgh (grant numbers EP/L016834/1 and EP/J015040/1). The first author would like to thank Chris Mower, Iordanis Chatzinikolaidis, and Hsiu-Chin Lin for the engaging and fruitful discussions, and to express special gratitude to Theodoros Stouraitis for the companionship and encouragement to write this piece of work. 


\section{REFERENCES}

[1] Farhad Aghili. Inverse and direct dynamics of constrained multibody systems based on orthogonal decomposition of generalized force. In IEEE International Conference on Robotics and Automation, ICRA, 2003. doi:10.1109/ROBOT.2003.1242217.

[2] Farhad Aghili. A unified approach for inverse and direct dynamics of constrained multibody systems based on linear projection operator: Applications to control and simulation. IEEE Transactions on Robotics, 21(5):834849, oct 2005. doi:10.1109/TRO.2005.851380.

[3] Farhad Aghili. Projection-based modeling and control of mechanical systems using non-minimum set of coordinates. In IEEE/RSJ International Conference on Intelligent Robots and Systems, IROS. IEEE, sep 2015. doi:10.1109/IROS.2015.7353815.

[4] Farhad Aghili and Jean Claude Piedbœuf. Simulation of motion of constrained multibody systems based on projection operator. Multibody System Dynamics, 10(1): 3-16, aug 2003. doi:10.1023/A:1024584323751.

[5] Leopoldo Armesto, João Moura, Vladimir Ivan, Mustafa Suphi Erden, Antonio Sala, and Sethu Vijayakumar. Constraint-aware learning of policies by demonstration. The International Journal of Robotics Research, 37(13-14):1673-1689, jul 2018. doi:10.1177/0278364918784354.

[6] K. S. Banerjee, C. Radhakrishna Rao, and Sujit Kumar Mitra. Generalized inverse of matrices and its applications. In Berkeley Symposium on Mathematical Statistics and Probability, 1973. doi:10.2307/1266840.

[7] Herman Bruyninckx and Oussama Khatib. Gauss' principle and the dynamics of redundant and constrained manipulators. IEEE International Conference on Robotics and Automation, ICRA, 3:2563-2568, 2000. doi:10.1109/ROBOT.2000.846414.

[8] Peter Corke. Robotics, Vision and Control: Fundamental Algorithms In MATLAB, Second Edition. Springer Publishing Company, Incorporated, 2017. doi:10.1007/9783-319-54413-7.

[9] Vincent De Sapio and Oussama Khatib. Operational space control of multibody systems with explicit holonomic constraints. In IEEE International Conference on Robotics and Automation, ICRA, 2005. doi:10.1109/ROBOT.2005.1570562.

[10] Vincent De Sapio, Oussama Khatib, and Scott Delp. Task-level approaches for the control of constrained multibody systems. Multibody System Dynamics, 16(1): 73-102, aug 2006. doi:10.1007/s11044-006-9017-3.

[11] Niels Dehio, Joshua Smith, Dennis Leroy Wigand, Guiyang Xin, Hsiu-chin Lin, Jochen J Steil, and Michael Mistry. Modeling and control of multi-arm and multi-leg robots: Compensating for object dynamics during grasping. In IEEE International Conference on Robotics and Automation, ICRA, 2018. doi:10.1109/ICRA.2018.8462872.
[12] Alexander Dietrich, Christian Ott, and Jaeheung Park. The hierarchical operational space formulation: Stability analysis for the regulation case. IEEE Robotics and Automation Letters, 3(2):1-1, 2018. doi:10.1109/LRA.2018.2792154.

[13] Keith L. Doty, Claudio Melchiorri, and Claudio Bonivento. A theory of generalized inverses applied to robotics. The International Journal of Robotics Research, 12(1):1-19, feb 1993. doi:10.1177/027836499301200101.

[14] Adrien Escande, Nicolas Mansard, and Pierre-Brice Wieber. Hierarchical quadratic programming: Fast online humanoid-robot motion generation. The International Journal of Robotics Research, 33(7):1006-1028, jun 2014. doi:10.1177/0278364914521306.

[15] Roy Featherstone. An empirical study of the joint space inertia matrix. The International Journal of Robotics Research, 23(9):859-871, sep 2004. doi:10.1177/0278364904044869.

[16] Roy Featherstone. Rigid Body Dynamics Algorithms. Springer-Verlag, 2007. doi:10.1007/978-1-4899-7560-7.

[17] Roy Featherstone. Exploiting sparsity in operationalspace dynamics. International Journal of Robotics Research, 29(10):1353-1368, jan 2010. doi:10.1177/0278364909357644.

[18] Roy Featherstone and Oussama Khatib. Load independence of the dynamically consistent inverse of the jacobian matrix. The International Journal of Robotics Research, 16(2):168-170, apr 1997. doi:10.1177/027836499701600203.

[19] Gene H. (Gene Howard) Golub. Matrix computations. The Johns Hopkins University Press, Baltimore, Md., fourth edition, 2013.

[20] Oussama Khatib. A unified approach for motion and force control of robot manipulators: The operational space formulation. IEEE Journal on Robotics and Automation, 3(1):43-53, feb 1987. doi:10.1109/JRA.1987.1087068.

[21] Oussama Khatib. Inertial properties in robotic manipulation: An object-level framework. The International Journal of Robotics Research, 14(1):19-36, feb 1995. doi:10.1177/027836499501400103.

[22] Hsiu-Chin Lin, Joshua Smith, Keyhan Kouhkiloui Babarahmati, Niels Dehio, and Michael Mistry. A projected inverse dynamics approach for dual-arm cartesian impedance control. In IEEE International Conference on Robotics and Automation, ICRA, 2018. doi:10.1109/ICRA.2018.8461202.

[23] Michael Mistry and Ludovic Righetti. Operational space control of constrained and underactuated systems. Robotics: Science and Systems, RSS, 2011. doi:10.15607/RSS.2011.VII.031.

[24] Jun Nakanishi, Rick Cory, Michael Mistry, Jan Peters, and Stefan Schaal. Operational space control: A theoretical and empirical comparison. The International Journal of Robotics Research, 27(6):737-757, jun 2008. 
doi:10.1177/0278364908091463.

[25] Valerio Ortenzi, Rustam Stolkin, Jeffrey A. Kuo, and Michael Mistry. Projected inverse dynamics control and optimal control for robots in contact with the environment: A comparison. In IEEE/RSJ International Conference on Intelligent Robots and Systems, IROS, 2015. doi:10.1109/IROS.2015.7353942.

[26] Jaeheung Park and Oussama Khatib. Contact consistent control framework for humanoid robots. In IEEE International Conference on Robotics and Automation, ICRA, 2006. doi:10.1109/ROBOT.2006.1641993.

[27] Jan Peters, Michael Mistry, Firdaus Udwadia, Rick Cory, Jun Nakanishi, and Stefan Schaal. A unifying methodology for the control of robotic systems. In IEEE/RSJ International Conference on Intelligent Robots and Systems, IROS, 2005. doi:10.1109/IROS.2005.1545516.

[28] Ludovic Righetti, Jonas Buchli, Michael Mistry, and Stefan Schaal. Inverse dynamics control of floating-base robots with external constraints: A unified view. In IEEE International Conference on Robotics and Automation, ICRA, 2011. doi:10.1109/ICRA.2011.5980156.

[29] Luis Sentis and Oussama Khatib. Control of free-floating humanoid robots through task prioritization. In IEEE International Conference on Robotics and Automation, ICRA, 2005. doi:10.1109/ROBOT.2005.1570361.

[30] Luis Sentis and Oussama Khatib. A whole-body control framework for humanoids operating in human environments. In IEEE International Conference on Robotics and Automation, ICRA, 2006. doi:10.1109/ROBOT.2006.1642100.

[31] Bruno Siciliano, Lorenzo Sciavicco, Luigi Villani, and Giuseppe Oriolo. Robotics: Modelling, Planning and Control. Springer Publishing Company, Incorporated, 2008. doi:10.1007/978-1-84628-642-1.

[32] Firdaus E. Udwadia and Robert E. Kalaba. Analytical Dynamics: A New Approach. Cambridge University Press, 1996. doi:10.1017/CBO9780511665479.

[33] Patrick M. Wensing, Luther R. Palmer, and David E. Orin. Efficient recursive dynamics algorithms for operational-space control with application to legged locomotion. Autonomous Robots, 38(4):363-381, apr 2015. doi:10.1007/s10514-015-9420-9.

[34] Haruo Yanai, Kei Takeuchi, and Yoshio Takane. Projection Matrices, Generalized Inverse Matrices, and Singular Value Decomposition. Springer New York, 2011. doi:10.1007/978-1-4419-9887-3. 